\title{
The challenges of managing cancer related venous thromboembolism in the palliative care setting
}

\author{
S Noble
}

Postgrad Med J 2007;83:671-674. doi: 10.1136/pgmi.2007.061622

Cancer patients with venous thromboembolism (VTE) pose particular management challenges since they have an increased risk of bleeding and recurrent thrombosis compared to the noncancer population. Also, as the disease progresses so do the hazards of anticoagulation, and patients in the palliative stages of their cancer could be viewed as a separate disease group with respect to diagnosis and management. As the focus from curative treatment moves towards symptom control, physicians face several challenges in providing the most appropriate care. Palliative care patients have rarely been included in research on VTE and the supporting evidence needs to be extrapolated cautiously. Quality of life aspects of VTE and their management may be a more appropriate outcome measure in this stage of disease than radiological end points. This paper looks at the challenges facing professionals in the management of VTE in the advanced cancer patient.

Correspondence to

Dr S Noble, Department of Palliative Medicine, Ward

B6N, Royal Gwent Hospital, Cardiff Rd, Newport, NP2O 2UB, UK; simon.noble@ gwent.wales.nhs.uk

Received 14 May 2007 Accepted 18 July 2007
$\mathrm{T}$ he association between venous thromboembolism (VTE) and cancer has been recognised since the 19th century and was first described by Armand Trousseau. ${ }^{1}$ Patients with cancer have a higher risk of developing deep vein thrombosis (DVT) and pulmonary embolus (PE) than the noncancer population due to secretion of cancer related procoagulants and an increased activation of coagulation factors such as tissue factor, thrombin anti-thrombin complex and factor VIIa. The prevalence of clinically apparent VTE in cancer patients is up to $15 \%$, yet as the cancer progresses so does the prothrombotic tendency. ${ }^{2}$ Studies in the advanced cancer population have shown an incidence of asymptomatic VTE in up to $52 \%$ of inpatients. ${ }^{3}$ This figure is similar to postmortem studies which show evidence of PE in $50 \%$ of cancer patients. ${ }^{4}$ The management of cancer associated VTE is different to that of the non-cancer population. Emerging evidence has demonstrated the advantages of low molecular weight heparin (LMWH) over warfarin with respect to VTE recurrence, drug-drug interactions, absorption and safety. ${ }^{6}$ Within the palliative care cancer population, the management of VTE needs to be based on quality of life as much as clinical efficacy. Available existing evidence, extrapolated from a healthier study population, needs to be applied in the context of a poor performance status, non-homogeneous patient group. To do this one needs to appreciate the fundamental challenges of managing cancer related VTE in the palliative care setting.

\section{CHALLENGE 1: DEFINING THE POPULATION}

Palliative care patients are not solely looked after by palliative care teams. The majority will come under the acute care of the admitting physician, surgeon or oncologist. Decision making for palliative care patients will need to weigh up the burden or benefit of investigations and treatments against the decision to treat solely symptomatically. Nevertheless, the nihilistic view that "a large PE might be a nice way to go" is not supported by the evidence. Cancer deaths from a PE are rarely sudden, quick and symptom-free. Postmortem studies of cancer patients who died of PE suggest most patients were symptomatic for an average of $2 \mathrm{~h}$ before death. ${ }^{4}$ In addition, symptoms due to fatal PE, such as dyspnoea, anxiety and pain, are difficult to remedy in the ensuing cardiovascular collapse and diminished perfusion which impairs the delivery of necessary symptom control medicines in the terminal stages.

The original model of palliative care, founded around the hospice movement, looked after patients at the end of life in a predominantly inpatient population. The model of palliative care has changed and many services integrate their involvement earlier in the patient's cancer journey when symptoms first become problematic. They will therefore be involved with patients of better performance status. There is no agreed definition of a palliative care patient although various suggestions have been made. ${ }^{7}$ Defining a patient as palliative, based on predicted prognosis, is one possibility-for example, accepting involvement of patients with an estimated prognosis $<6$ months. However, doctors are known to be over optimistic in their estimation of survival and this method would lead to referrals too late in life. ${ }^{8}$ In addition, patients are living longer with advanced disease and consequently have symptom control needs for longer.

Another possible definition is based on the presence of metastatic disease which would suggest that the cancer was incurable. In practice, as oncology develops more treatments for patients with metastases, they will remain under the main care of oncology and may do so for years. Considering the World Health Organization's

Abbreviations: CTPA, computed tomographic pulmonary angiography; DVT, deep vein thrombosis; INR, international normalised ratio; LITE, Long-term Innohep Treatment evaluation; $\mathrm{LMWH}$, low molecular weight heparin; $\mathrm{PE}$, pulmonary embolus; SPCU, Specialist palliative care unit; VTE, venous thromboembolism; V/Q scan, ventilation/ perfusion lung scintigraphy 
definition of palliative care, the speciality looks after "patients facing problems associated with life threatening illness". By this definition, not every patient with terminal disease needs palliative care and the service will focus on those with the most complex needs.

For the purpose of this paper a palliative care patient will be defined as someone with incurable cancer whose specialist care is predominantly under the care of the palliative care service. This definition, although not perfect, defines a heterogeneous group of varied performance status, prognosis and degree of metastatic disease. It will include those who, despite having liver or brain metastases, may still have good performance status, as well as those who are asymptomatic but of performance status 3 or 4 .

\section{CHALLENGE 2: RECOGNITION OF THE VTE}

Despite a suggested incidence of asymptomatic VTE in over $50 \%$ of palliative care patients, the reported incidence is considerably smaller at around $1-5 \%{ }^{9}$ There may be several reasons for this. First, the true natural history of asymptomatic VTE in the palliative care patient is not known. It is possible that not all thrombi will progress to symptoms and, therefore, cause no problems. Secondly, the reported incidence may represent an under appreciation of the risk factors of advanced malignancy among palliative care physicians. The practice of VTE management varies worldwide and over $25 \%$ of British oncologists were unaware of the associated risks of VTE with cancer. ${ }^{10}{ }^{11}$ Finally, the common presenting symptoms of VTE are similar to those of other common pathologies experienced by patients with advanced cancer-for example, a painful swollen leg raises a high index of suspicion in a non-cancer patient while there are several causes to consider in the palliative care setting (box 1). Likewise dyspnoea, the most common symptom associated with PE, is seen in many common conditions associated with advanced cancer (box 2). It is important to recognise that although many conditions may mimic VTE, the presence of one such diagnosis does not negate the concurrent diagnosis of thrombosis. Acute conditions such as infection, heart failure and local venous obstruction will increase the prothrombotic state and likelihood of undiagnosed VTE. ${ }^{12}$

\section{CHALLENGE 3: CONFIRMATION OF VTE Use of D-dimers}

Plasma D-dimers are specific cross-linked fibrin derivatives produced when fibrin is degraded by plasmin. Concentrations are therefore raised by thrombolysis, making them a highly sensitive indicator for VTE. Although sensitive for VTE, high Ddimer concentrations also occur in infection, malignancy and postoperative states, thus being insufficiently specific for making a positive diagnosis. ${ }^{13} 14$

D-dimer values are now widely recognised as being an important exclusary test for the diagnosis of VTE, with a negative predictive value of close to $100 \%$. However, their use in cancer patients is limited since they may have increased concentrations due to their underlying malignant disease. They currently have no role in the diagnosis of VTE in the palliative care setting.

\section{Confirmation of suspected DVT}

There are a number of techniques available for the detection of lower limb DVT. Each has advantages and disadvantages including different degrees of sensitivity, specificity, operator dependence, cost, accessibility and invasiveness. Radiological diagnosis will depend upon local practice and availability of investigations.

Doppler ultrasonography is the most widely available, cheap, non-invasive test and has largely replaced venography as the first choice for symptomatic DVT. A recent meta-analysis suggested the sensitivity of ultrasonography to be $89 \%$ overall
Box 1: Common causes of swollen legs in the palliative care patient

Unilateral

- Deep vein thrombosis

- Cellulitis

- Nodal disease in groin

- Lymphoedema

Bilateral

- Deep vein thrombosis

- Hypoalbuminaemia

- Heart failure

- Medicines - for example, steroids, nifedipine

- Lymphoedema

- Pelvic disease causing reduced venous oufflow

\section{Box 2: Common causes of dyspnoea in advanced} cancer

- Pneumonia

- Pulmonary oedema

- Pleural effusion

- Anaemia

- Lung metastases

- Lymphangitis

- Muscle fatigue

- Concurrent pulmonary illness

- chronic obstructive pulmonary disease

- emphysema

- interstitial lung disease

- congestive cardiac failure

for symptomatic DVT and 97\% for above knee thrombus. Problems with ultrasonography include a poor sensitivity for asymptomatic disease, difficulties in diagnosing DVT recurrence, and limited visualisation in the pelvis. ${ }^{15}{ }^{16}$

Impedance plethysmography is also commonly used but has similar limitations in the setting of recurrent thrombosis, asymptomatic DVT and DVT below the knee or in the pelvis. ${ }^{15} 16$

Venography is the reference standard diagnostic test but has in large been replaced by non-invasive tests. In practice it is the most reliable test for the diagnosis of asymptomatic thrombus and thrombus located within the calf or pelvis. However, this involves injecting intravenous contrast and has largely been replaced by less invasive tests in most hospitals. ${ }^{15-17}$

\section{Confirmation of suspected pulmonary embolus}

Confirmation of suspected PE first requires the diagnosis to be considered. Secondly the clinician must decide whether a positive diagnosis of PE would alter the patient's management. In a specialty where the balance of quality of life and active treatment is paramount, one must consider the burden of obtaining radiological confirmation which may need to be done on a site away from the specialist palliative care unit (SPCU). In addition to considering the burden of diagnosis on the patient, one must also consider whether the treatment of a confirmed PE is safe and feasible. The anticoagulation of someone 
at high risk of haemorrhage would be inappropriate, as would someone who is entering the terminal phase of their condition, where other symptom control measures could be instigated. It is clear that decision making must therefore take into account quality of life aspects of treatment and investigation and individualisation of care which considers the patient's wishes.

Imaging tests available for the diagnosis of $\mathrm{PE}$ include ventilation/perfusion lung scintigraphy (V/Q scan), pulmonary angiography, and spiral computed tomographic pulmonary angiography (CTPA) (table 1). Unfortunately all of the imaging techniques have limitations and access to each may vary between SPCUs.

Pulmonary angiography remains the reference standard but is technically demanding and invasive. ${ }^{18}{ }^{19}$ For this reason it is unlikely to be the investigation of choice in the palliative care setting, unless the patient is robust enough to undergo the test. It is also associated with a major complication rate of $0.5 \%$ and a mortality rate of $0.1 \%$.

V/Q scans are relatively simple to perform and non-invasive, but when used in isolation can be non-diagnostic in up to $70 \%$ of suspected PEs. ${ }^{20}$ Within the advanced cancer population, concurrent lung pathology makes interpretation even more challenging. Better results have been obtained combining clinical probability with V/Q scan interpretation, but its role in the palliative care setting is likely to be limited. ${ }^{21}$

CTPA is gaining wider acceptance and popularity since it is readily available in most hospitals and is well tolerated by patients. Although this technique is excellent for diagnosing central or lobar PEs, it is unable to rule out isolated subsegmental PEs. Meta-analyses suggest CTPA has a sensitivity of about $70 \%$ and a specificity of $90 \%$ in the diagnosis of $\mathrm{PE}^{22}$ For this reason a negative result does not exclude a diagnosis in $30 \%$ of patients. Despite these limitations, CTPA is likely to be the most appropriate diagnostic tool in the investigation of $\mathrm{PE}$ in the palliative care setting.

\section{CHALLENGE 4: TREATMENT OF VTE}

Although warfarin is the mainstay of long term anticoagulation for VTE, several studies have demonstrated significant increases in rates of bleeding among cancer patients receiving oral anticoagulation (as high as $21.6 \%$ in one study). ${ }^{23}{ }^{24}$ The bleeding risk in palliative care patients is even greater. An audit of anticoagulation in a Scottish SPCU reported 15 bleeding episodes in 17 patients, which was improved to 11 episodes in 18 patients by stringent international normalised ratio (INR) monitoring. ${ }^{25}$ Although these numbers are small, other palliative care physicians share these findings and there is a tendency to maintain the INR at subtherapeutic levels in advanced cancer. ${ }^{9}$

In addition to an increased risk of bleeding, cancer patients are more likely to develop further thrombotic events on warfarin than non-cancer patients. ${ }^{18} 22$ Studies have shown that up to $27 \%$ of cancer patients will develop secondary VTE despite therapeutic warfarin administration, and the incidence is likely to be higher in advanced malignancy since:

- patients become more pro-thrombotic as the malignancy advances

- patients tend to be anticoagulated at subtherapeutic levels to minimise the risk of bleeding.

There is increasing evidence to suggest that LMWH is the drug of choice in the long term management of VTE. ${ }^{26}$ One qualitative study suggests that $\mathrm{LMWH}$ is an acceptable intervention in the palliative care setting and, for some patients, preferable to warfarin. ${ }^{27} \mathrm{LMWH}$ has been shown to have several benefits over warfarin in cancer patients, which are listed below.
Table 1 Imaging tests available for the diagnosis of pulmonary embolus

\begin{tabular}{llll}
\hline Investigation & $\begin{array}{l}\text { Accuracy in } \\
\text { diagnosing PE }\end{array}$ & $\begin{array}{l}\text { Burden on } \\
\text { patient }\end{array}$ & $\begin{array}{l}\text { Usefulness in } \\
\text { palliative care }\end{array}$ \\
\hline Pulmonary angiography & ++++ & +++ & ++ \\
V/Q scan & ++ & + & + \\
CTPA & ++ & + & ++ \\
\hline
\end{tabular}

CTPA, computed tomographic pulmonary angiography; PE, pulmonary embolus; V/Q scan, ventilation/perfusion lung scintigraphy

- Dose is calculated according to patient weight and so there is no need to monitor anticoagulation

- Efficacy is not altered by changes in nutritional status

- Not affected by absorption problems or poor oral intake

- Efficacy not altered by new medicines.

Although several trials have addressed long term treatment for VTE with oral anticoagulant versus LMWH, only three have looked at patients with cancer. Meyer and associates randomised patients with cancer and VTE to 3 months of treatment with either LMWH enoxaparin $(1 \mathrm{mg} / \mathrm{kg})$ or warfarin. ${ }^{28}$ The composite outcome of major bleeding and recurrent VTE was observed in 15 out of $71(21.1 \%)$ patients receiving warfarin, compared to 7 of the $67(10.5 \%)$ receiving LMWH $(p=0.09)$. The Long-term Innohep Treatment evaluation (LITE) trial randomised 200 patients with acute VTE and cancer to receive either unfractionated heparin followed by warfarin for 84 days at a targeted INR of 2.5, or the LMWH tinzaparin (175 IU/kg) for 85 days. ${ }^{29}$ The rate of recurrent VTE at 3 months was $6 \%$ in the LMWH group compared to $10 \%$ in the warfarin group, and at 1 year $7 \%$ and $16 \%$, respectively $(p=0.044)$.

Lee and associates reported the results of the CLOT trial, which was a large multicentre trial comparing treatment with the LMWH dalteparin with oral anticoagulant treatment in patients with active cancer presenting with acute VTE. ${ }^{26} \mathrm{~A}$ total of 338 patients were enrolled into each arm and were well matched for gender, age, outpatient treatment and performance status. Twenty-seven patients in the LMWH group experienced recurrent VTE compared with 53 in the oral anticoagulant group. Patients receiving long term LMWH treatment had a significantly lower cumulative risk of recurrent VTE at 6 months than those who received long term oral anticoagulant treatment $(8.8 \%$ vs $17.4 \%, 52 \%$ risk reduction; $\mathrm{p}=0.0017$ ). Major bleeding was seen in $19 / 338$ (5.6\%) patients receiving LMWH compared with $12 / 338$ (3.6\%) in the oral anticoagulant group $(\mathrm{p}=0.27)$. Corresponding data for any bleeding were $13.6 \%$ and $18.5 \%$, respectively $(\mathrm{p}=0.09)$.

These three studies clearly establish a case for LMWH as the first line treatment of cancer related VTE, and the acknowledged hazards of warfarin in palliative care support its use in the advanced cancer patient. The CLOT study excluded patients of performance status 3 or 4, while the LITE and Myers study populations included $47 \%$ and $50 \%$ patients with metastatic disease, respectively. Myers recorded that $40 \%$ of patients were no longer receiving active treatment.

Although no studies have been conducted specifically in the palliative care environment, one prospective cohort study in patients with metastatic cancer (brain or liver) and VTE investigated the effect of using low dose dalteparin. Two hundred and three patients received a 7 day course of dalteparin according to body weight followed by a fixed dose of 10000 IU dalteparin once daily for at least 3 months. ${ }^{30}$ Eleven patients (5.4\%) developed major bleeding complications ( six fatal) during the 3 month study period, and 18 patients $(8.9 \%)$ developed VTE recurrences (two patients died). There 


\section{Learning points}

- Venous thromboembolism (VTE) is common in advanced cancer patients.

- The management of VTE in this patient group needs to take into account the impact of investigations and treatments on their overall quality of life.

- Involvement of patients in the decision making process is essential.

- Anticoagulated patients are at high risk of bleeding complications and recurrent thromboses.

- Low molecular weight heparins are more efficacious and have a better impact on quality of life than warfarin in the advanced cancer patient.

- Warfarin is not advised in the treatment of VTE in the advanced cancer patient.

were no higher complication rates in patients with either liver or brain metastases.

\section{CHALLENGE 5: HOW LONG TO CONTINUE ANTICOAGULATION?}

In the non-cancer population, anticoagulation should be continued for 3-6 months. ${ }^{31}$ This is based on the clinical knowledge that the prothrombotic risk factor that precipitated the initial event (stasis, surgery, etc) has now resolved and cessation of anticoagulation will have no sequelae. The prothrombotic tendency, in the advanced cancer patient, will by definition be ongoing and, if anything, increase as the disease progresses. None of the cancer VTE studies were continued past 6 months so there are no clinical trial data supporting anticoagulation past this period of time. However, a recent case series of 62 palliative care patients with VTE reported patients receiving LMWH for up to 243 days. Interestingly, of the seven patients who had their anticoagulation stopped at 6 months, three (43\%) had recurrence of their thrombus and recommenced anticoagulation. If one is to consider anticoagulation long term, there will still come a time when the continuation of LMWH offers no symptomatic benefit or improvement in quality of life. In the case series reported, most patients continued anticoagulation until they entered the last few days of life. ${ }^{32}$ These data, while shedding light on how long we should continue anticoagulation, also suggest that the majority of palliative care patients are appropriate for investigation and treatment of suspected VTE. Sometimes the biggest danger faced by the palliative care patient is not a complication of the disease process but rather the paternalistic nihilism inflicted on them by the attending clinician.

\section{CONCLUSION}

While the evidence favouring the use of LMWH versus warfarin in the management of VTE is encouraging, there is a need for a robust clinical study in the palliative care population to look at the best way to treat and prevent VTE. In particular, a comparison of the CLOT protocol with the lower dose regimen described by Monreal is clearly needed. Careful consideration will need to be given to measurable end points that are relevant to the advanced cancer population. In a patient group where time is precious, quality of life outcomes are as important as radiological ones.

Competing interests: None

\section{REFERENCES}

1 Trousseau A. Phlegmasia alba dolens. In: Clinique Medicale d'Hotel-Dieu de Paris, vol 3. Paris: JB Balliere et Fils, 1865:654-812.

2 Bick RL. Alterations of haemostasis with malignancy. Semin Thromb Hemost 1978;5:1-26.

3 Johnson MJ, Sproule MW, Paul J. The prevalence and associated variables of deep venous thrombosis in patients with advanced cancer. Clin Oncol 1999; 11:105-10.

4 Ambrus JL, Ambrus CM, Pickren JW. Causes of death in cancer patients. J Med 1975:6:61-4

5 Sproul EE. Carcinoma and venous thrombosis: the frequency of association of carcinoma in the body or tail of the pancreas with multiple venous thrombosis. Am J Cancer 1938;34:566-85.

6 Lee AY. Management of thrombosis in cancer: primary prevention and secondary prophylaxis. Br J Haematol 2005;128:291-302.

7 Maltoni M, Caraceni A, Brunelli C, et al. Prognostic factors in advanced cancer patients: evidence-based clinical recommendations - a study by the Steering Committee of the European Association for Palliative Care. J Clin Oncol 2005;23:6240-8

8 Christakis NA, Lamont EB. Extent and determinants of error in doctors' prognoses in terminally ill patients: prospective cohort study. BMJ 2000;320:469-73.

9 Johnson MJ, Sherry K. How do palliative physicians manage venous thromboembolism? Palliat Med 1997;11:462-8.

10 Wolf RA. Are patients with cancer receiving adequate thromboprophylaxis? Results from FRONTLINE. Cancer Treat Rev 2003;29(Suppl 2):7-9.

11 Kirwan CC. Prophylaxis for venous thromboembolism during treatment for cancer: questionnaire survey. BMJ 2003;327:597-8.

12 Turpie AG. Thrombosis prophylaxis in the acutely ill medical patient: insights from the prophylaxis in MEDical patients with ENOXaparin (MEDENOX) trial. Am J Cardiol 2000;86(12B):48M-52M.

13 Kelly J, Hunt BJ. Role of d-dimers in diagnosis of venous thromboembolism. Lancet 2002;359:456-8.

14 Kelly J, Rudd A, Lewis RR, et al. Plasma D-dimers in the diagnosis of venous thromboembolism. Arch Intern Med 2002;162:747-56.

15 Kearon C, Julian JA, Newman TE, et al. Noninvasive diagnosis of deep venous thrombosis. McMaster Diagnostic Imaging Practice Guidelines Initiative. Ann Intern Med 1998;128:663-77

16 Fraser JD, Anderson DR. Deep venous thrombosis: recent advances and optimal investigation with U.S. Radiology 1999;211:9-24.

17 Rose SC, Zwiebel WJ, Nelson BD, et al. Symptomatic lower extremity deep venous thrombosis: accuracy, limitations and role of color duplex flow imaging in diagnosis. Radiology 1990;175:639-44.

18 Nilsson T, Carlsson A, Mare K. Pulmonary angiography: a safe procedure with modern contrast media and technique. Eur Radiol 1998;8:86-9.

19 Hudson ER, Smith TP, McDermott VG, et al. Pulmonary angiography performed with iopamidol: complications in 1,434 patients. Radiology 1996;198:61-5.

20 The PIOPED Investigators. Value of the ventilation/perfusion scan in acute pulmonary embolism. Results of the prospective investigation of pulmonary embolism diagnosis (PIOPED). JAMA 1990;263:2753-9.

21 Hull RD, Hirsh J, Carter CJ, et al. Diagnostic value of ventilation-perfusion lung scanning in patients with suspected pulmonary embolism. Chest 1985;88:819-28

22 Remy-Jardin M, Remy J, Deschildre F, et al. Diagnosis of pulmonary embolism with spiral CT: comparison with pulmonary angiography and scintigraphy. Radiology 1996;200:699-706.

23 Hutten BA, Prins MH, Gent $M$, et al. Incidence of recurrent thromboembolic and bleeding complications among patients with venous thromboembolism in relation to both malignancy and achieved international normalized ratio: a retrospective analysis. J Clin Oncol 2000;18:3078-83.

24 Prandoni P. Antithrombotic strategies in patients with cancer. Thromb Haemost 1997;78(suppl): 141-4.

25 Johnson MJ. Problems of anticoagulation within a palliative care setting: an audit of hospice patients taking warfarin. Palliat Med 1997;11:306-12.

26 Lee AY, Levine M, et al. Low-molecular-weight heparin versus a coumarin for the prevention of recurrent venous thromboembolism in patients with cancer. N Engl J Med 2003;349:146-53.

27 Noble SIR, Finlay IG. Is long-term low molecular weight heparin an acceptable to palliative care patients in the treatment of cancer related venous thromboembolism? A qualitative study. Palliat Med 2005;19:197-201.

28 Meyer G, Marjanovic Z, Valcke J, et al. Comparison of low-molecular-weight heparin and warfarin for the secondary prevention of venous thromboembolism in patients with cancer. Arch Intern Med 2002;162:1729-35.

29 Hull RD, Pineo GF, Mah AF, for the LITE Study Investigators. Long-term lowmolecular-weight heparin versus usual care in proximal-vein thrombosis patients with cancer. Am J Med 2006;1 19:1062-72.

30 Monreal M, et al. Fixed-dose low-molecular-weight heparin for secondary prevention of venous thromboembolism in patients with disseminated cancer: a prospective cohort study. J Thromb Haemost 2004;2:1311-5.

31 British Thoracic Society. Guidelines for the management of suspected acute pulmonary embolism. Thorax 2003;58:470-84.

32 Noble SI, Hood K, Finlay IG. The use of long-term low-molecular weight heparin for the treatment of venous thromboembolism in palliative care patients with advanced cancer: a case series of 62 patients. Palliat Med 2007;21:473-6. 